\title{
Explosive fragmentation of liquids in spherical geometry
}

\author{
A. Milne ${ }^{1,4}$ - A. Longbottom ${ }^{1}$ - D. L. Frost ${ }^{2}$ - J. Loiseau ${ }^{2}$. \\ S. Goroshin ${ }^{2}$ - O. Petel $^{3}$
}

Received: 8 July 2015 / Revised: 27 May 2016 / Accepted: 30 May 2016

(C) The Author(s) 2016. This article is published with open access at Springerlink.com

\begin{abstract}
Rapid acceleration of a spherical shell of liquid following central detonation of a high explosive causes the liquid to form fine jets that are similar in appearance to the particle jets that are formed during explosive dispersal of a packed layer of solid particles. Of particular interest is determining the dependence of the scale of the jet-like structures on the physical parameters of the system, including the fluid properties (e.g., density, viscosity, and surface tension) and the ratio of the mass of the liquid to that of the explosive. The present paper presents computational results from a multimaterial hydrocode describing the dynamics of the explosive dispersal process. The computations are used to track the overall features of the early stages of dispersal of the liquid layer, including the wave dynamics, and motion of the spall and accretion layers. The results are compared with new experimental results of spherical charges surrounded by a variety of different fluids, including water, glycerol, ethanol, and vegetable oil, which together encompass a significant range of fluid properties. The results show that the number of jet structures is not sensitive to the fluid properties, but primarily dependent on the mass ratio. Above a certain mass ratio of liquid fill-to-explosive burster $(F / B)$, the number of
\end{abstract}

Communicated by F. Zhang.

A. Milne

alec.milne@ fluidgravity.co.uk

1 Fluid Gravity Engineering Ltd., St. Andrews, Fife KY16 9NX, UK

2 Mechanical Engineering Department, McGill University, Montreal, QC H3A 0C3, Canada

3 Mechanical and Aerospace Eng., Carleton University, Ottawa, ON K1S 5B6, Canada

4 Mathematical Institute, University of St. Andrews, St. Andrews, Fife KY16 9SS, UK jets is approximately constant and consistent with an empirical model based on the maximum thickness of the accretion layer. For small values of $F / B$, the number of liquid jets is reduced, in contrast with explosive powder dispersal, where small $F / B$ yields a larger number of particle jets. A hypothetical explanation of these features based on the nucleation of cavitation is explored numerically.

Keywords Experiment $\cdot$ Modelling $\cdot$ Explosion Fragmentation

\section{Introduction}

Detonation of a central high explosive charge, surrounded by a shell of material is a common practical geometry in many application areas, where the physics of the interaction of the detonation with the surrounding material continues to be studied. These areas include blast mitigation, fuel-air explosions, metalized explosives, and reactive casings. In this paper, we consider a shell of liquid surrounding an explosive charge in spherical geometry.

The behaviour following the detonation of a high explosive charge underwater has been studied in detail for many years (see for example, Cole [1]). A detonation in deep water can be considered as the case of a shell of water of infinite thickness, and in these conditions, one observes the growth and oscillation of a stable gas bubble. There have been many examples of this phenomenon and we illustrate this behaviour in Fig. 1.

In Fig. 1, one can see the detonation followed by an initial expansion, where some surface structure is observed, which may correspond to charge case effects, but at later times, one can observe the smooth and stable gas globe which is the characteristic of this phase of an underwater explosion. 
Fig. 1 Underwater bubble resulting from detonation of a spherical $10 \mathrm{~g}$ aluminized explosive charge in thin plastic case $(2 \mathrm{~cm}$ outer diameter) in a cylindrical tank at various times (left to right, top to bottom)

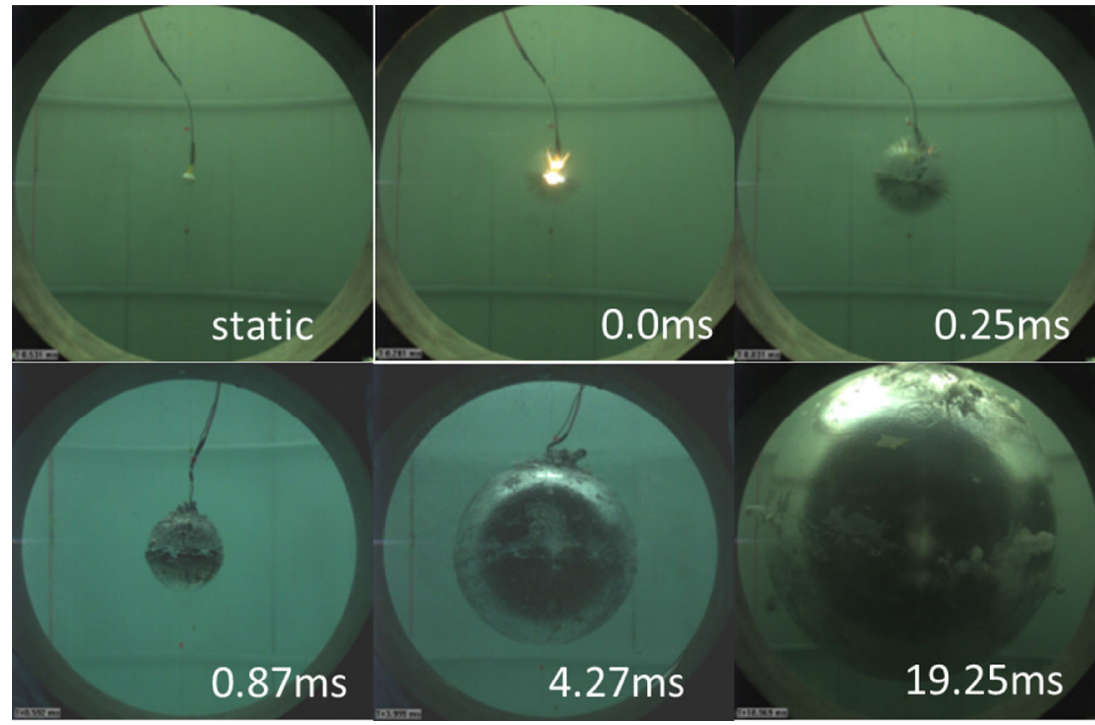

If one considers a shell of water with a finite thickness, the introduction of an outer boundary changes the stability of the system. In this case, the expansion is no longer stable and the shell forms jet-like structures, suggesting strongly that wave interactions from the outer boundary play a key role in the observed break-up process.

Milne et al. [2,3] have noted that during the dispersal of both liquid and particle layers, experimental evidence from a wide range of sources shows that the expanding cloud of explosively disseminated material is comprised of "particles" or fragments which have significantly different dimensions from those associated with the original material. Photographic evidence shows characteristic jets or fingers behind these spherically diverging fragments. Examples of the formation of these jet structures are given, for example, in Frost et al. [4] and Ripley and Zhang [5], or in Frost and Zhang [6] in the context of jet formation from heterogeneous blast explosives. Zhang et al. [7] have recently revisited old data to study particle jetting instabilities and hypothesise that charge casing may play an important role. This is an important study area in its own right but is beyond the scope of the current paper, where we deliberately use thin-walled glass cases. The explosive shock wave causes the thin-walled glass casing to fracture into very fine fragments which are carried out by the gas flow behind the shock. Previous work [8] has shown that these glass fragments are visible on flash X-ray radiographs taken during the explosive dispersal of solid particles. While the fracture of the glass into fine fragments could generate fine-scale perturbations at the liquid interface, the size of the glass fragments appears to be much less than the spacing of the subsequent liquid jets.

Further X-ray analysis of these systems has allowed Milne et al. [3] to show that the fragmentation process occurs early in the expansion, around the time that the release wave from the outer boundary reaches the inner boundary of the surrounding material. It is natural to consider the possibility that the breakup into jets is a result of classical hydrodynamic interchange instabilities (see Chandrasekhar [9] for discussion of and introduction to well-known examples, such as Rayleigh-Taylor, Richtmyer-Meshkov, or KelvinHelmholtz), and there is a large body of continuing research in this field (see, for example, Mankbadi and Balachandar [10] and references therein). Milne et al. [3] reported that direct numerical simulation of the experimental systems they studied showed that the growth rate of these instabilities was too slow to explain the growth observed by X-ray analysis, so other potential mechanisms were investigated. They showed that, for a given experiment, the numbers of fragments observed in the early-time radiographs and in the late-time photographic evidence are in good agreement. A simple model to predict the number of jets from powders was proposed, where the typical size of the fragment is determined by the thickness of the compressed layer at the time that the release wave from the outer boundary reaches the inner boundary of the surrounding material.

Explosive acceleration of a liquid shell differs from the corresponding situation with solid particles due to the presence of voids in the unperturbed particle bed. In the latter case, the shock propagating through the particles causes considerable compaction, and depending on the ductility of the particles, the compacted bed may approach a fully densified state and have considerable strength after compaction. In the case of shock loading of a liquid, the reflection of an expansion wave at the liquid surface and subsequent tension applied to the bulk liquid will lead to the cavitation of void growth and subsequent breakup of the bubbly liquid into a droplet aerosol [11]. 
Explosive dispersal of a liquid progresses through several regimes. In the first regime, which has been termed the ejection regime [12], the forces associated with the propagation of the shock wave through the liquid and rarefaction that returns from the liquid surface dominate the motion of the fluid. This is followed by a transition regime in which the explosive forces are comparable to aerodynamic forces on the liquid. In the final regime, the shock forces have decayed, and the motion of liquid droplets is governed by aerodynamic drag and droplet breakup is due to the competition between inertial and surface tension forces.

The present numerical work is concerned with the nearfield ejection regime which occurs on a timescale of hundreds of microseconds for charges with mass of the order of $1 \mathrm{~kg}$, where shock forces dominate. The present experimental work measures the jet structure in the late-time regime. Of particular interest is determining the dependence of the scale of the jet-like structures on the physical parameters of the system, including the fluid properties (e.g., density, viscosity, and surface tension) and the ratio of the mass of the liquid to that of the explosive. The number of liquid jets that are formed and the rate of droplet shedding and subsequent evaporation of the liquid will determine the size of the liquid-vapour-air cloud that is ultimately formed, which is of interest if the liquid is combustible.

In the present paper, the dynamics of the explosive dispersal process of a liquid is investigated both experimentally and with a multi-material hydrocode. The computations are used to track the features of the process, including the wave dynamics, and motion of the spall and accretion layers. A working hypothesis for dynamic liquid breakup is proposed in which the maximum thickness of the accretion layer determines the number of jets. This hypothesis is examined in light of experimental results with spherical charges containing a variety of different fluids to determine the influence of fluid properties. Water was chosen as a baseline fluid and other liquids were chosen that have significant variations in fluid properties, including glycerol (similar density and surface tension to water, but much higher viscosity), vegetable oil (similar density to water, but higher viscosity and lower surface tension), and ethanol (similar density and viscosity to water, but lower surface tension). Prior to the presentation of the experimental and computational results, existing theoretical models describing the dynamic fragmentation of liquids will first be considered.

\section{Theoretical models of liquid breakup}

\subsection{Mott theory}

The natural starting point for the breakup of liquid shells is Mott theory [13] (see also Grady [14] for a detailed review) which is widely used to model explosive fragmentation of solid shells. The theory builds on the assumption of a large number of randomly distributed crack nucleation sites which communicate via elastic or plastic waves. A consequence of this is a distribution of fragment sizes from very small to very large. When we examine the experimental data from radiographs and high-speed videos (e.g., [2,3] and this work), we observe a different size distribution which appears to be more monomodal in nature with no observable small fragments. This feature can be seen from typical video stills at late times in the expansion when the jets have come to rest, as shown in Figs. 3 and 9 below. If there was a wide range of fragment sizes, one would expect to see a wide range of jet lengths, but we observe a tight distribution of lengths suggesting a similar distribution of primary fragment sizes. We shall consider this observation in more detail below, but for the present, we note that this has led us to consider other models of liquid breakup.

The dynamic breakup of liquids has been considered theoretically by Grady [15]. He found that energy-limited spall in liquids was dominated by surface energy effects at low strain rates and by viscous dissipation at high rates. He also noted that there exists relatively little experimental information for liquid spall. His model can be used to estimate the order of magnitude of the liquid fragment size which in turn can determine the number of jets for a given volume of fluid.

The Grady model predicts fragment sizes in two limits, namely, surface tension dominated and viscous dissipation dominated. Which mechanism dominates is determined by the spall strength. As both surface tension and viscosity can act to stabilize the breakup of the other mode, the argument is that the dominant mechanism in any situation will be the one with the largest spall strength.

\subsection{Effect of surface tension}

For surface tension dominated problems, the Grady model predicts the fragment size $s$

$s=\left(\frac{48 \gamma}{\rho \dot{\varepsilon}^{2}}\right)^{1 / 3}$,

where $\gamma$ is the surface tension, $\rho$ is the density, and $\dot{\varepsilon}$ is the local strain rate.

\subsection{Effect of viscosity}

For viscous dominated problems, the Grady model gives fragment size $s$

$s=\left(\frac{8 \eta}{\rho \dot{\varepsilon}}\right)^{1 / 2}$

where $\eta$ is the viscosity. 


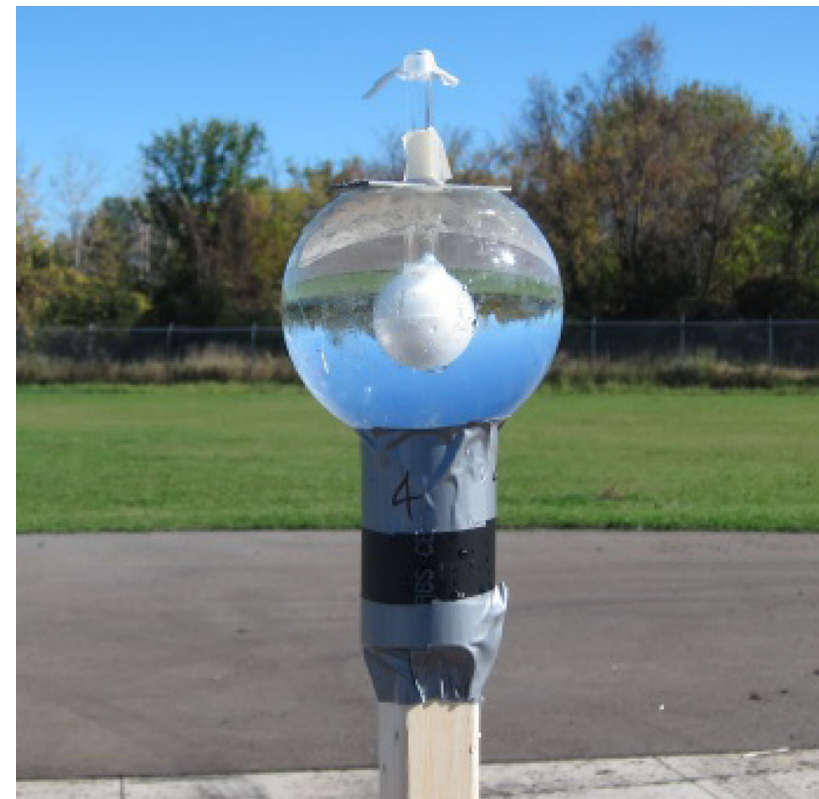

Fig. 2 Photograph of a $1 \mathrm{~L}$ glass sphere containing distilled water. Central burster contains $28 \mathrm{~g} \mathrm{C} 4$

The implications of the theoretical model predictions will be considered by comparing the breakup of liquids that have a large difference in viscosity, i.e., water and glycerol, in the next section.

\section{Experimental results}

Experiments were carried out with three different sized spherical glass casings with volumes of about 2.8, 1.0, and $0.4 \mathrm{~L}$, respectively. The largest casing was a thick-walled ( $3 \mathrm{~mm}$ thick) glass sphere, whereas the smaller two charges consisted of thin-walled ( $1 \mathrm{~mm}$ thick) glass spheres prepared by removing the filaments from commercial globe-style incandescent light bulbs. A photograph of a $1 \mathrm{~L}$ sphere is shown in Fig. 2, which also shows the central explosive burster consisting of $\mathrm{C} 4$ within a hollow plastic sphere (3 $\mathrm{mm}$ wall thickness). A tube attached to the burster sphere was used to allow the insertion of the detonator into the C4. Two different sized burster charges were used, either 5.1 or $3.8 \mathrm{~cm}$ outer diameter, containing either 80 or $28 \mathrm{~g}$ of $\mathrm{C} 4$, respectively. We consider a range of liquid fills (water, glycerol, ethanol, vegetable oil, and sodium polytungstate) chosen to have different initial densities, viscosities, and surface tensions to allow us to compare against the Grady models.

\subsection{Comparison of water and glycerol in spheres}

We begin by considering some experimental data for water and glycerol before going on to more detailed experimental and numerical analysis. The images shown in Fig. 3 were for 80 g C4 explosive surrounded by $2.8 \mathrm{~L}$ of liquid contained in a glass sphere. The water was at a density of $1000 \mathrm{~kg} / \mathrm{m}^{3}$ and the glycerol at a density of $1260 \mathrm{~kg} / \mathrm{m}^{3}$. This gives fillto-burster mass ratios $(F / B)$ of 34.5 and 40.9 , respectively. In later sections, we shall report the results of varying this ratio and the fill liquid.

As reported in [4], we find that counting jets directly from the image can be subjective. Intensity levels can strongly influence the interpretation. Since the experimental images show the jets to be uniformly distributed over a sphere, we prefer to scan the image along a radius (see Fig. 3) to obtain line plots of intensity and count features from these. Since the jet structures are much wider than a single pixel scan line, we have to take care in choosing representative scans. An arbitrary radial line will miss some structures, so we choose lines which show the largest number of radial features to analyse. This method still has a degree of subjectivity, but we find it to be the most consistent method. A comparison of the results of using different techniques for counting jets is given in an earlier publication [3]. Using this method gives the typical comparison (intensity vs. pixel number), as shown in Fig. 4.

This analysis suggests that there is roughly the same number of structures in each scan. There are similar highfrequency details in both signals, but the higher intensity (number of bits) in the glycerol data makes them more distinct. One can thus conclude from this data that the number of jets is comparable for these two liquids with between 11 and 14 radial features $(12+2 /-1)$ for the water data and between 10 and 13 radial features $(11+2 /-1)$ for the glycerol data. These numbers imply primary fragment sizes of order $7 \mathrm{~mm}$ in diameter.

\subsection{Comparison with Mott and Grady models}

We can use the scan data to measure typical widths of the structures and then plot a probability density distribution function for the number of features in a given bin size of widths. Figure 5 shows (in blue) a measured fragment number distribution function from our video data for water. As noted above, this does not have the features of a Mott distribution function (shown in red). We do not observe any small scale features. This may in part be due to the lack of resolution in our basic data, but we see similar distributions for different resolutions. We would definitely expect to see the tail of a Mott distribution clearly, but we do not. Since [3] noted that the number of features seen in early X-ray data was also seen in late-time video data, we include a probability density function obtained by measuring the widths of features in scans of X-ray data for a sand experiment (shown in green). The two measured functions from different measuring systems and at different times are remarkably similar and are more akin to a monomodal than a Mott distribution. 
Fig. 3 Single frames taken from high-speed video of explosive dispersal of spherical charges containing a water and b glycerol. In each case, the charge volume was $2.8 \mathrm{~L}$ with a central burster of about $80 \mathrm{~g} \mathrm{C} 4$. The thick red line from the centre to the edge is a typical radial line along which we take line plots of intensity for more detailed analysis
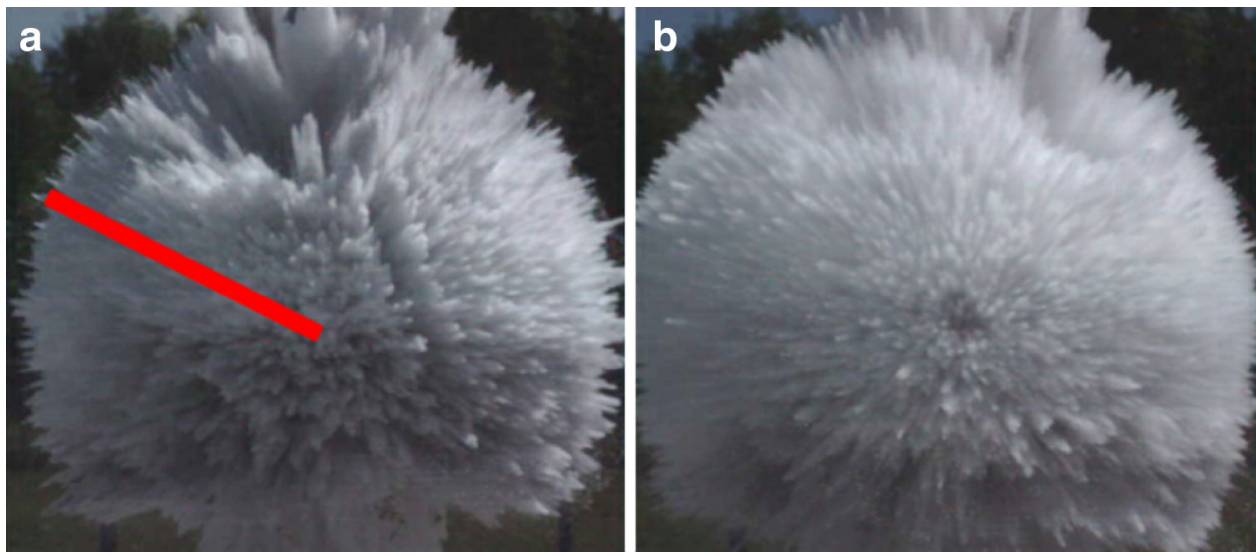

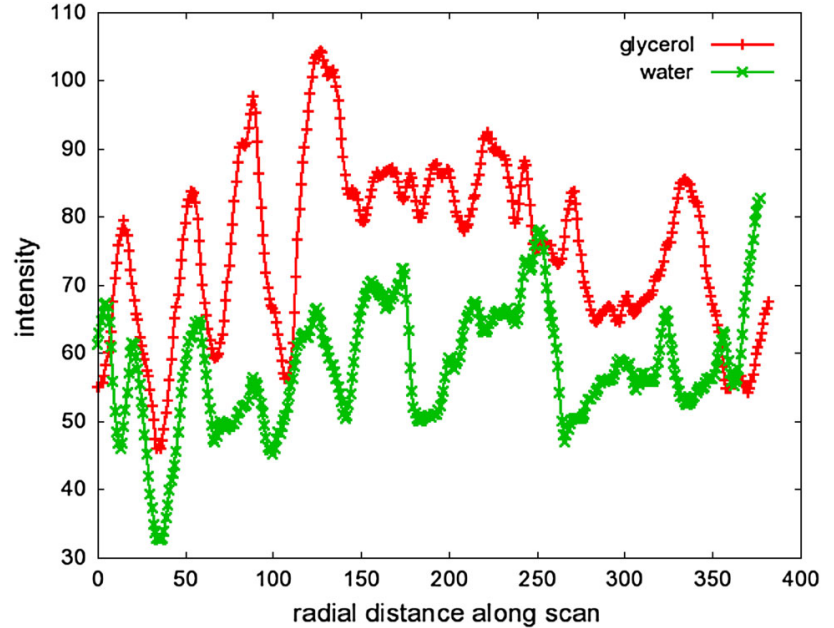

Fig. 4 Comparisons of scans of intensity vs. radius in pixel number units from the images of Fig. 3

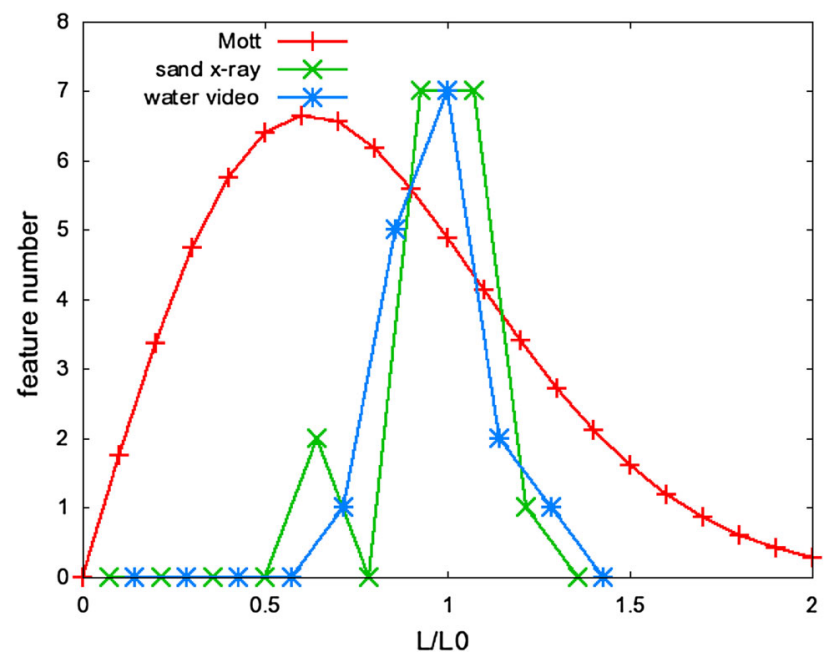

Fig. 5 Comparisons of probability distribution functions of the number of features of a given size (normalised against a mean size L0) from the Mott theory, analysis of scans of water video data from this work, and scans from sand shell X-rays from [3]
We are interested to know what determines this typical size of feature (and associated number of jets).

We can now return to the Grady model in light of the experimental results. For surface-tension-dominated liquid breakup, (1) gives a prediction of the fragment size. For water, one typically has ambient values of $\gamma=0.07 \mathrm{~J} / \mathrm{m}^{2}$ and $\rho=1000 \mathrm{~kg} / \mathrm{m}^{3}$. For glycerol in these experiments, $\rho=1260 \mathrm{~kg} / \mathrm{m}^{3}$, but water and glycerol have very similar values of surface tension (or surface energy) making them natural materials to consider experimentally. For these ambient values, applying the Grady model to our typical experimental conditions suggests that $s=0.6 \mathrm{~mm}$ which is significantly smaller than the dimensions implied by our current analysis of this class of experiments, where we see much larger primary fragments (roughly an order of magnitude larger), but we should note that the ambient value of surface tension may not be representative of shocked liquid.

At high strain rates with high viscosity, viscous terms dominate the spall strength making the liquid more resistant to spall. For ambient conditions, water has $\eta=10^{-3} \mathrm{Pas}$, while for glycerol, $\eta=1.5 \mathrm{Pas}$. These values give predictions for the viscous dominated fragment size from (2) of $s=46 \mu \mathrm{m}$ for water, and $s=1.3 \mathrm{~mm}$ for glycerol, suggesting we should see differences in our experiments if viscosity has a major influence. We should note that viscosity increases significantly in shocked liquids, so the absolute values of fragment size quoted above will be underestimates.

If one steps back and only considers the trends implied by the Grady model (since we are uncertain of absolute values for the shocked liquids), these suggest that the glycerol fragments should be roughly twice the size of the water fragments, and thus, one should have approximately four times more water jets than glycerol jets (or twice as many features along a radial scan for water). This is at odds with the observations which showed a similar number of features.

If viscosity alone dominates, then since (at room temperature) glycerol viscosity is $\sim 1500$ times higher than water, one should expect $\sqrt{1500}$ (or about 40 times) larger parti- 
cles (or about 40 times fewer jets). This is not observed in the experiments. The evidence is thus that viscosity is not a dominant effect.

The above arguments led us to consider a series of experiments using different liquids in different sizes of container and with a range of $F / B$ ratios.

\subsection{Effect of scale}

Our experimental series considers different sizes of container and different charge sizes. If surface tension and viscosity are not major influences on fragmentation, then one might expect to have hydrodynamic scaling, so that one would obtain the same number of fragments for different charges that have similar $F / B$ ratios. In Fig. 6, we compare the results from two such experiments.

One can see very similar numbers of features in both experiments, so our numerical and experimental analysis will use $F / B$ as our independent variable. Figure 7 shows some typical scans of images for different liquids in the same container using the same charge, so that $F / B$ only varies with fill density (and here varies between 27 and 41).

We use the above class of data to determine the number of radial features and to estimate error bars. The results will be plotted and compared with numerical predictions in some figures below.

\section{Numerical models}

It has been previously noted [2,3] that for powders surrounding an explosive charge, the number and size of primary fragments correlate well with the thickness of the compressed

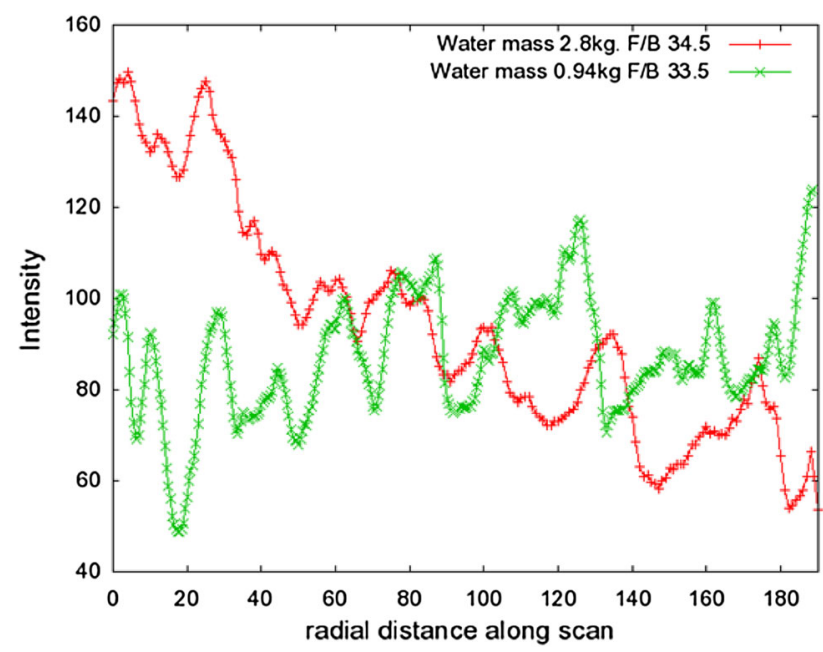

Fig. 6 Intensity vs. radial distance for two water experiments at different scales (water mass of 0.94 and $2.8 \mathrm{~kg}$ ) but with similar values of $F / B$

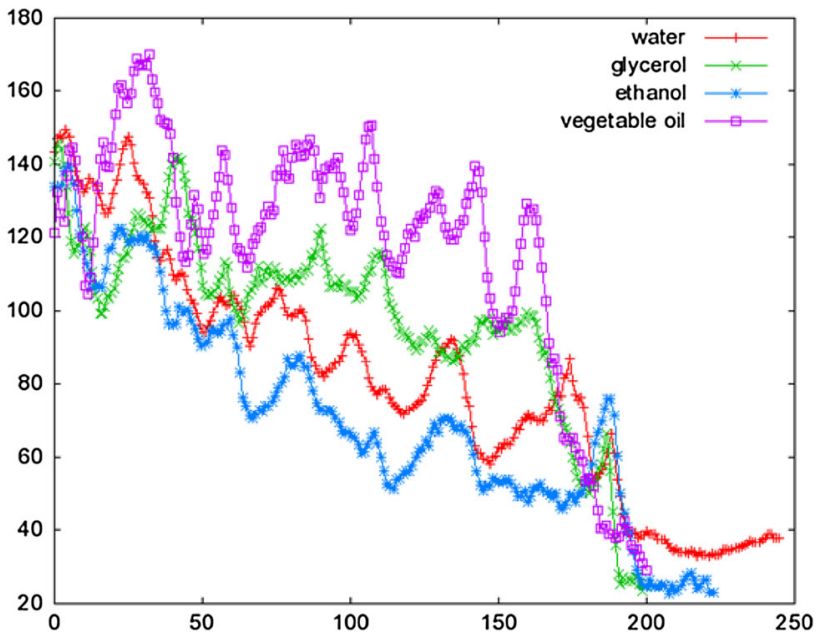

Fig. 7 Representative scans of spherical shots with different liquids in the same size of container with the same size of charge

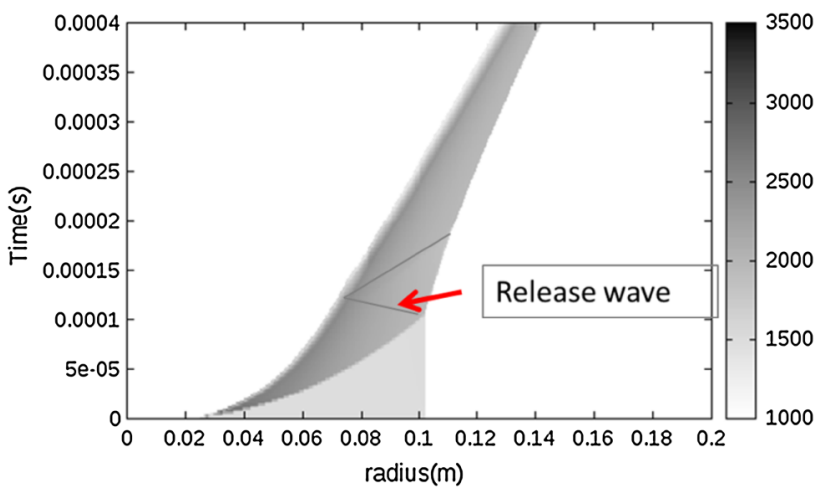

Fig. 8 Calculated powder density vs. time and radius for glass beads surrounding a $\mathrm{C} 4$ explosive in spherical geometry at $F / B=80$ to illustrate wave and interface behaviour in the powder. The scale for the density is shown to the right of the plot, in units of $\mathrm{kg} / \mathrm{m}^{3}$

layer at the time of a release wave reaching the inner surface (see Fig. 8).

Radius-time plots (of the type shown in Fig. 8) can be used to identify the thickness $\delta$ of the compressed layer when the release wave reaches the inner surface and the mean shell radius $r$ at this time. If one assumes that the primary breakup phase occurs at this time and further assume, for simplicity, that these fragments are cubes, then the number of fragments predicted is

$N=4 \pi\left(\frac{r}{\delta}\right)^{2}$.

Since we have chosen to use the number of features along a radial scan $n$ as our experimental measure, it is useful to record how this measure relates to the number of fragments predicted. By assuming a constant solid angle for each feature, we find 
Fig. 9 Glass bead dispersal at various ratios of $F / B$
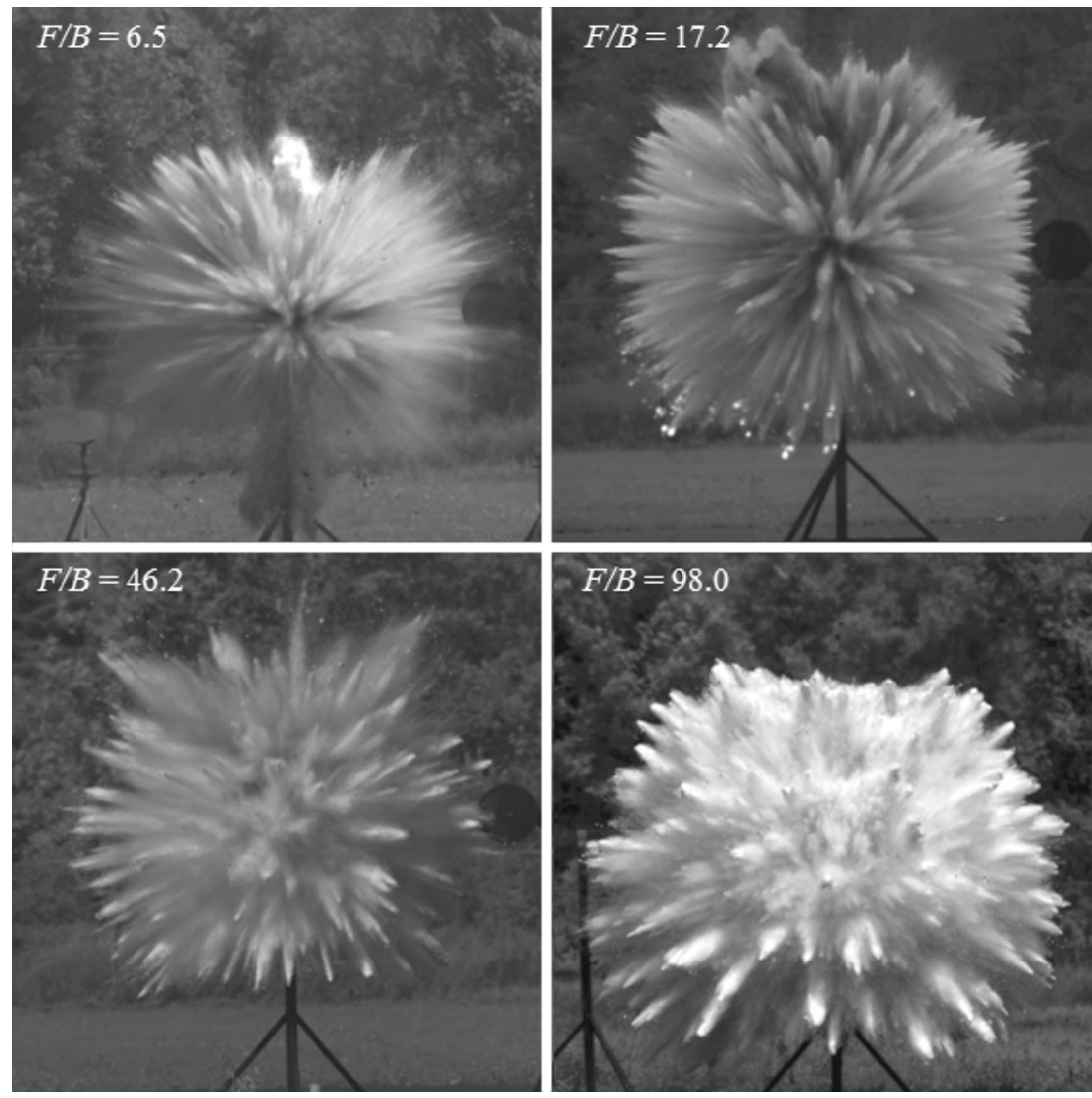

$n=\sqrt{\frac{\pi N}{16}}$.

In this work, we will consider how these observables vary with fill-to-burster mass ratio $F / B$.

To illustrate the expected behaviour, let us consider the limit of thin shells, purely for mathematical simplicity, since in many of our experiments, this becomes a poor approximation. In this limit, the charge outer radius and the typical shell radius are approximately the same $(r)$. In this case, the burster mass

$B \sim r^{3}$

and the fill mass

$F \sim r^{2} \delta$

One can see that the number of features along a radial line will be expected to decrease with $F / B$, that is

$n \propto \frac{1}{(F / B)}$.

Thus, one expects the number of features to decrease with increasing $F / B$ for powders. We observe this to be the case as illustrated by the examples for glass bead fills below

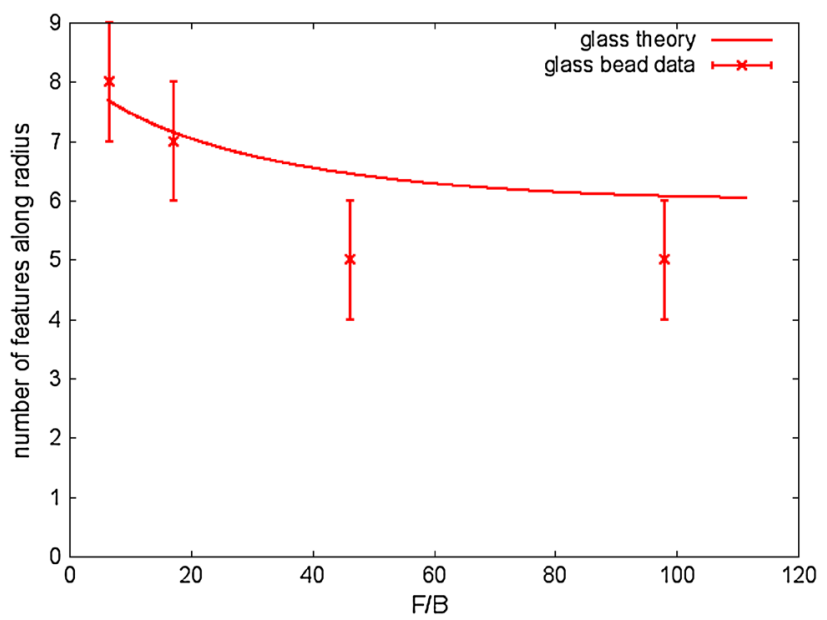

Fig. 10 Measured number of features (with error bars) along a radial line for glass bead dispersion at various ratios of $F / B$ along with theory using (5)

(Fig. 9). These images suggest that the number of jets is decreasing with $F / B$. We use our preferred method of counting features along a radial scan to quantify this in Fig. 10.

For liquids, there is a slightly more complex wave structure [1]. As shown in Fig. 11, liquids exhibit a spall layer and a low-density cavitation region forms between this and the inward propagating release wave. At the time that the release 


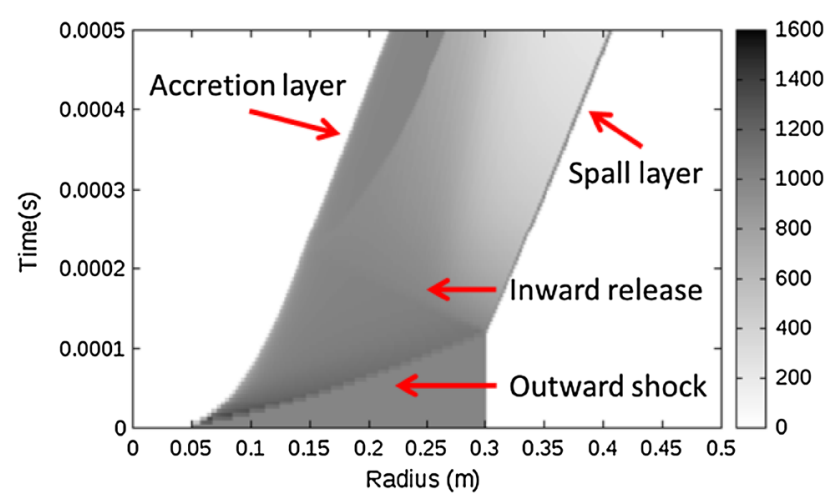

Fig. 11 Calculated liquid density vs. time and radius for water surrounding a $\mathrm{C} 4$ explosive in spherical geometry to illustrate wave and interface behaviour in the liquid (here, $F / B=130$ )

wave reaches the inner surface, in the one-dimensional calculation, all the liquid behind the spall layer has cavitated. The thickness of the shell at this time is thus an unlikely dimension for the observed dense primary liquid fragments.

A plausible alternative dimension to consider as typical of the primary fragment size is the maximum thickness of the accretion layer (the layer of higher density water that builds up, as the spalled water is re-compressed during the expansion). The maximum occurs as a balance between thickening through the recompression of the cavitated region and thinning by spherical divergence.

If, as argued above, we are eliminating surface tension and viscosity, the main remaining difference between water and glycerol is density. As an extreme case, one can consider just changing the density in a numerical model. Using the universal liquid Hugoniot [16] allows one to model different liquids easily and self-consistently. We assume a minimum pressure of zero to model the lack of support of tension in a liquid. We systematically investigate the effects of varying density and sound speed in a range of geometries in the remainder of this paper.

\subsection{Model details}

Since the experimental data shows that the same numbers of features are seen at different scales, assuming that the $F / B$ ratio remains constant, we choose to model a range of $F / B$ values and material types. We use the universal liquid Hugoniot as our equation of state, which requires a reference density and a sound speed to be provided (Table 1).

The sound speed for the specific vegetable oil used was not measured, but the past work with liquid edible oils [17] indicates that the sound speed is comparable to that of water.

As a baseline modelling problem, we consider a charge radius of $0.5 \mathrm{~cm}$ and vary the outer radius of the fill from 1
Table 1 Density and sound speed data used in equations of state for experimental liquids

\begin{tabular}{lcl}
\hline Material & Density $\left(\mathrm{kg} / \mathrm{m}^{3}\right)$ & Sound speed $(\mathrm{m} / \mathrm{s})$ \\
\hline Water & 1000 & 1460 \\
Ethanol & 789 & 1180 \\
Glycerol & 1260 & 1920 \\
Vegetable oil & 915 & 1460 \\
Sodium polytungstate & 2820 & 1405 \\
\hline
\end{tabular}

Table 2 Geometries used and resulting $F / B$ ratios for water

\begin{tabular}{llr}
\hline Material & Outer radius $(\mathrm{m})$ & $F / B$ \\
\hline Water & 0.01 & 4.37 \\
Water & 0.015 & 16.25 \\
Water & 0.02 & 39.37 \\
Water & 0.025 & 77.5 \\
\hline
\end{tabular}

to $2.5 \mathrm{~cm}$. The table below illustrates the range of values of $F / B$ resulting for water (Table 2).

We use the calculated radius vs. time plots of this data to identify the maximum thickness, $\delta$, of the accretion layer and the mean shell radius, $r$, at this time. If one assumes that the primary break-up phase occurs at this time and further assume, for simplicity, that these fragments are cubes, then the number of fragments predicted is given by (4) and the number of features along a radial scan is given by (5) above.

\subsection{Water results}

Figure 12 shows the number of features along a radial scan predicted by modelling using the hypothesis that the primary fragment dimension is equal to the maximum thickness of the accretion layer. As we increase $F / B$, this model predicts that the number of features along a scan (and thus the total number of jets) will decrease. This is the same trend as was previously seen in both model and experiment for powder fills.

In this figure, we also plot the result of our analysis of the experimental data and our error bar estimates. The numbers that are observed are similar to those predicted by this hypothesis, but the shape of the curve is not matched. The experiment shows that there are fewer (as opposed to more) jets at low values of $F / B$. We repeat the analysis for ethanol, glycerol, vegetable oil, and sodium polytungstate, and the results are shown in Fig. 13. In all these cases, we see results similar to those found for water. The shape of the curve from modelling in all cases differs from the experimental curve. 


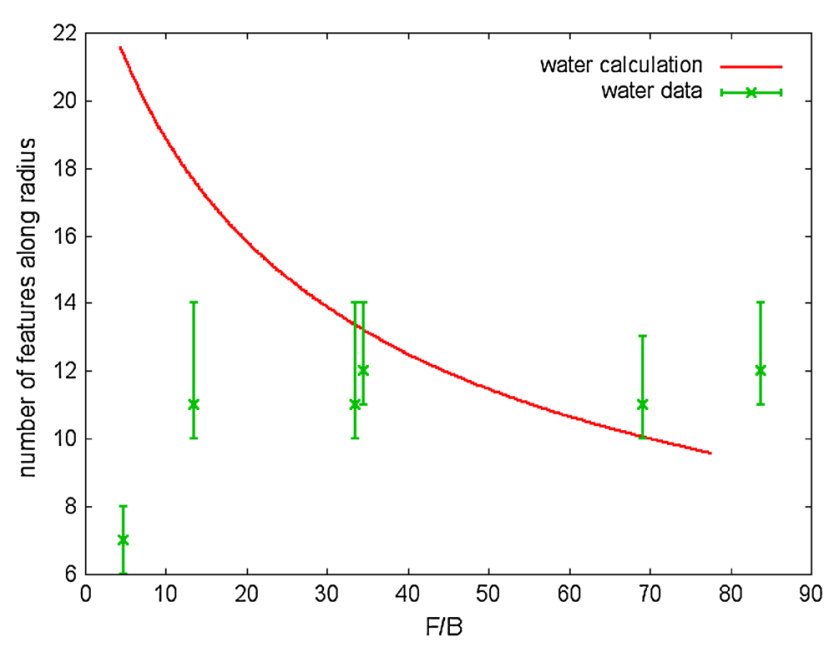

Fig. 12 Predicted and measured number of features along a radial line for water as a function of $F / B$

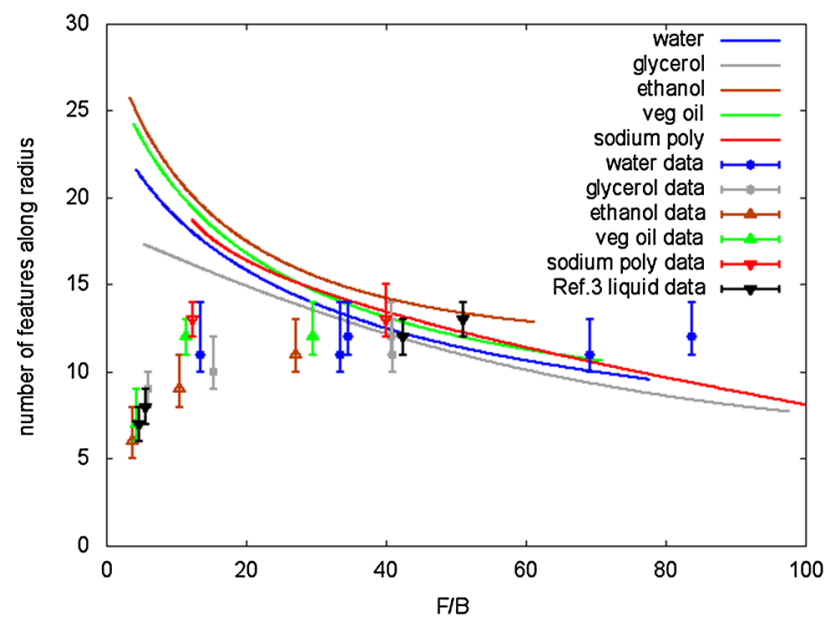

Fig. 13 Predicted and measured number of features along a radial line for all liquids considered in this paper as a function of $F / B$

\section{Discussion}

It is useful at this stage to plot all the modelling results and experimental data in one figure. In Fig. 13, regardless of material, one sees the same basic behaviour for all liquids, both experimentally and numerically, but the underlying curves differ between experiment and theory.

For large $F / B(>15)$, experiment suggests a very flat line for liquids at $F / B$, so that the number of jets is independent of both liquid and $F / B$. The model predictions are similar in number at high $F / B$, but show a pronounced decrease in features as $F / B$ increases over the whole range. All the liquids show the opposite trend at the lower end, i.e., the number of jets decreases as the $F / B$ ratio decreases. In Fig. 13, we also show some data from experiments performed using water fills at AWE and reported in [2,3]. The data lies on the same curve, even though size and the confining case material are very different.

\section{Analysis}

Using (5), we convert the measured number of features along a radial line to the total number of jets in the sphere. When one plots this number against $F / B$ (Fig. 14), one can see two regimes. At low $F / B$, the number of jets appears to be linearly dependent on $F / B$. At high $F / B$, the number tends to a constant value independent of $F / B$. These results are consistent with hydrodynamic scaling of the break-up mechanism and the fact that strain rate is an important parameter. All the liquids considered in this paper lie on the same lines.

In [3], we noted that the radiographs for powder fills suggested that the primary break-up mechanism showed the features of brittle fracture (with cracks forming and defining the fragment edges). Numerical modelling showed qualitative agreement with this mechanism.

When one looks at the radiographs for water as reported in [2], one observes (e.g., in Fig. 16) that the primary fragments appear to be liquid ligaments surrounding low-density regions. This suggests that a cavitation as opposed to a cracking mechanism is at play. We will use numerical modelling to further investigate this possibility by testing a potential hypothesis based on the nucleation of cavitation regions. If we build on the observed hydrodynamic scaling and further assume that the number of nucleation sites for cavitation is constant per-unit volume, we can explore whether these two properties can explain the two straight line regions of Fig. 14. Figure 15 shows that, at low $F / B$, the number of ligaments between cavitation cells is proportional to the number of cavitation regions and thus will grow with $F / B$ in the same

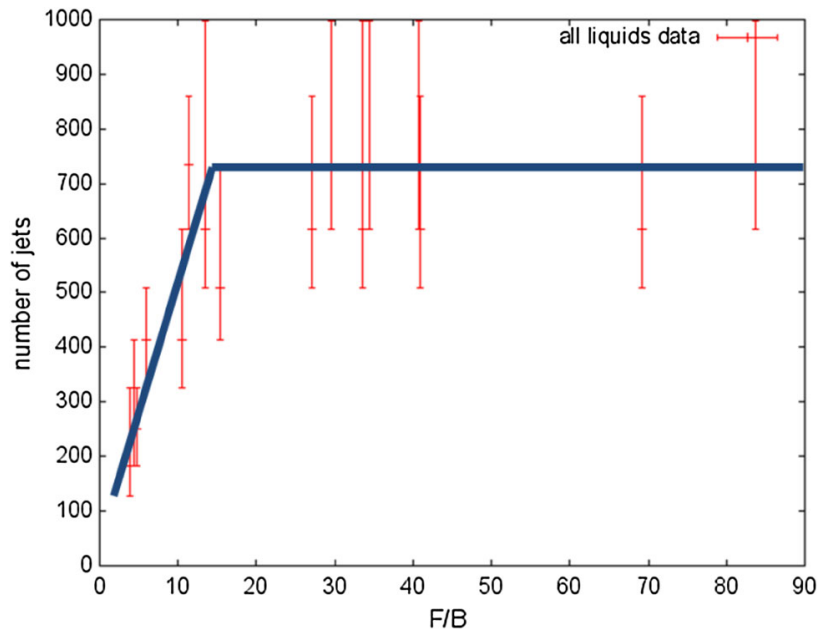

Fig. 14 Measured number of jets in the sphere for all liquids considered in this paper as a function of $F / B$ with two straight line fits 


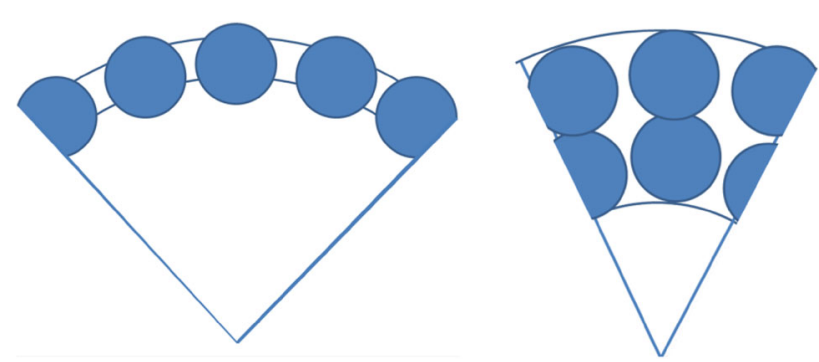

Fig. 15 Schematic showing cavitation geometry at low $F / B$ (left) and high $F / B$ (right). Filled blue circles represent growing cavitation regions in a conical section of the spherical shell. Gaps between cavitation regions are hypothesized as locations of ligaments observed in radiographs

manner as the number of nucleation sites for cavitation. The assumption of a constant number of sites per-unit volume leads to a straight line region at low $F / B$. At high $F / B$, there can be more than one cavitation region in the radial direction. The cavitation zones might be expected to join up, so that the number of ligaments or liquid regions between them is no longer proportional to the number of nucleation sites, but might be expected to saturate at the number of cavitation zones in the inner layer. This will lead to an approximately constant number of liquid regions which we take to be the origins of the later jets. Going one step further allows one to note that the intersection of the two straight lines shown in Fig. 14 identifies the case where the average distance between nucleation points is equal to the thickness of the shell at the break-up time.

As in [3], we consider a three-dimensional model of the detonation and expansion. In this case, we randomly seed the initial conditions with nucleation points (here, small volumes of partially cavitated liquid) separated by an average distance determined by the intersection of the curves in Fig. 14.

Figure 16 compares the result of this calculation for water at $F / B=4$ (the low region) with a radiograph from the experimental configuration reported in [2]. The features near the centre line of the experimental data are due to the joints required to assemble the two component hemispheres of the experimental system. We do not model this feature in our calculation.

As well as the accretion layer and spall layer introduced earlier, both calculation and experiment show fine structure. Light regions (which we interpret as ligaments of liquid) surround darker and thus lower density regions (which we interpret as cavitated regions). One can see good qualitative agreement between the two results.

Figure 17 compares the result of a calculation for water at $F / B=39$ (the high region) with a radiograph. Once again one can see good qualitative agreement between the two results adding a strong degree of self-consistency to the view that cavitation is the origin of the early structure in radi-

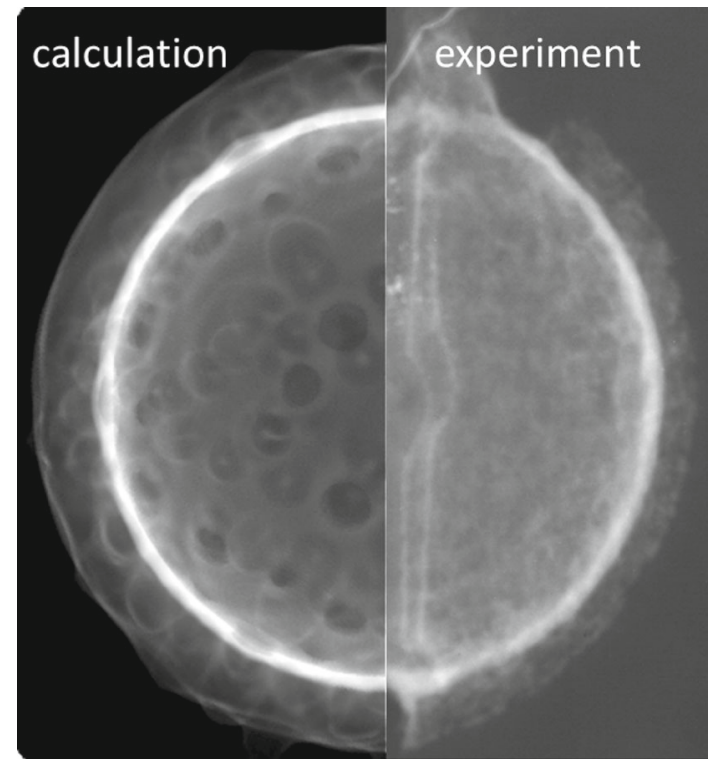

Fig. 16 Calculated result (left $)$ and experimental radiograph (right) for explosive dispersal of water at $F / B=4$. Brighter regions correspond to higher mass per-unit area in the X-ray beam

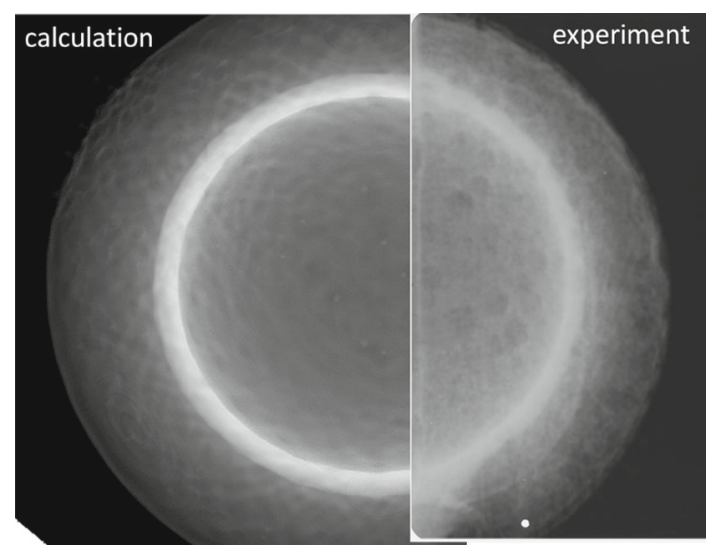

Fig. 17 Calculated result (left) and experimental radiograph (right) for explosive dispersal of water at $F / B=39$. Brighter regions correspond to higher mass per-unit area in the $\mathrm{X}$-ray beam

ographs of liquid shells. We note that the calculation is only a simulation, not a prediction of the experiment, since the number of nucleation points has been chosen from the counting analysis of the experimental data. We also note that this model only addresses the early stages of the expansion and has not considered any detail of the experimentally observed late-time jet formation

\section{Conclusions}

We have reported here an experimental and numerical study aimed at investigating the mechanisms which determine the number of jets seen in explosive dissemination of liquids. 
When there is no outer boundary (e.g., an underwater explosion at depth), one observes an extremely stable gas bubble with no jetting. When one introduces an outer boundary, one observes jetting, so we clearly no longer have a stable expansion of the system, suggesting strongly that wave interactions from the outer boundary play a key role in the observed breakup process. We thus sought to investigate in more detail the causes of the observed jetting.

We observed that the distribution of sizes of jets or primary fragments did not fit with a Mott distribution in that no large fragment tail or small scale features were observed. We thus considered the theoretical models for breakup of bulk liquids due to Grady. His early work noted that there was a lack of experimental data to test against and this provided one motivation for our work. The independent variables in these models were density, viscosity, and surface tension (as well as strain rate) which are very amenable to variation in liquids. Our results show trends which do not match the Grady model predictions, suggesting that some other mechanisms dominate in our problem.

We have previously studied explosive dissemination of powders (where the independent variables for Grady models are harder to prescribe experimentally) and have found good empirical correlations with primary fragment size (and thus number of jets) and predicted shell thickness. The shell structure in liquids is more complex due to the presence of a spall layer, so we considered an amended empirical model here (using the maximum thickness of the spall layer as our measure). This measure predicts more jets in liquids than in powders (as observed) and gives reasonable estimates for the number of jets at high $(>15)$ values of $F / B$, but the underlying shape of the curve of $n$ vs. $F / B$ does not match experiments which (unlike the case of powders) shows fewer jets at low $F / B$.

If one plots all of the data from our chosen range of liquids with differing initial surface tensions, viscosities, and densities on one graph of measured number of features along a radius against $F / B$, one sees a common curve. Since experiments were performed at a range of length scales, this shows that hydrodynamic scaling applies. This also suggests that the properties varied in the experiments are not dominant in determining primary fragment size. We note that experiments with very different case materials (glass and plastic) also lie on this curve, so we have no evidence that case effects are dominating. Using spherical symmetry to convert this curve into a plot of total number of jets vs. $F / B$ gives two straight line regions. We hypothesise that these two curves are consistent with a cavitation mechanism, where the number of nucleation sites per-unit volume is a constant. When we perform three-dimensional calculations based on this number of nucleation sites, we get good qualitative agreement with the shape and number of the features observed in experimental radiographs. Prediction (as opposed to post-shot simulation) of the experimental behaviour will require more knowledge of the causes of the initial nucleation sites for cavitation and more work is planned along these lines.

Open Access This article is distributed under the terms of the Creative Commons Attribution 4.0 International License (http://creativecomm ons.org/licenses/by/4.0/), which permits unrestricted use, distribution, and reproduction in any medium, provided you give appropriate credit to the original author(s) and the source, provide a link to the Creative Commons license, and indicate if changes were made.

\section{References}

1. Cole, R.H.: Underwater Explosions. Princeton University Press, Princeton (1948)

2. Milne, A.M., Parrish, C., Worland, I.: Dynamic fragmentation of blast mitigants. Shock Waves 20, 41-51 (2010)

3. Milne, A.M., Floyd, E., Longbottom, A.W., Taylor, P.: Dynamic fragmentation of powders in spherical geometry. Shock Waves $\mathbf{2 4}$, 501-513 (2014)

4. Frost, D.L., Gregoire, Y., Petel, O., Goroshin, S., Zhang, F.: Particle jet formation during explosive dispersal of solid particles. Phys. Fluids 24, 091109 (2012)

5. Ripley, R.C., Zhang, F.: Jetting instability mechanisms of particles from explosive dispersal. J. Phys. Conf. Ser. 500, 152012 (2014)

6. Frost, D.L., Zhang, F.: The nature of heterogeneous blast explosives. In: Proceedings of the 19th International Symposium on Military Aspects of Blast and Shock, 1-6 October, Calgary (2006)

7. Zhang, F., Ripley, R.C., Yoshinaka, A., Findlay, C.R., Anderson, J., von Rosen, B.: Large-scale spray detonation and related particle jetting instability phenomenon. Shock Waves 25, 239-254 (2015)

8. Frost, D.L., Ornthanalai, C., Zarei, Z., Tanguay, V., Zhang, F.: Particle momentum effects from the detonation of heterogeneous explosives. J. Appl. Phys. 101(11), 113529 (2007)

9. Chandrasekhar, S.: Hydrodynamic and Hydromagnetic Instability. Clarendon Press, Oxford (1961)

10. Mankbadi, M., Balachandar, S.: Multiphase effects on spherical Rayleigh-Taylor instability. Phys. Fluids 26, 023301 (2014)

11. Kedrinski, V.K.: Hydrodynamics of Explosion: Experiments and Models. In: Shock Wave and High Pressure Phenomena series. Springer, Berlin (2005)

12. Gardner, D.R.: Near-field dispersal modeling for liquid fuel-air explosives, SAND90-0686 report (1990)

13. Mott, N.F.: Fragmentation of shell cases. Proc. R. Soc. A 189, 300-308 (1947)

14. Grady, D.: Fragmentation of Rings and Shells: The Legacy of N.F. Mott. Springer, Berlin (2006)

15. Grady, D.E.: The spall strength of condensed matter. J. Mech. Phys. Solids 36(3), 353-384 (1988)

16. Woolfolk, R.W., Cowperthwaite, M., Shaw, R.: A "universal" Hugoniot for liquids. Thermochim. Acta 5, 409-414 (1973)

17. Coupland, J.N., McClements, D.J.: Physical properties of liquid edible oils. J. Am. Oil Chem. Soc. 74, 1559-1564 (1997) 